\title{
Software Solutions for Harmonic Conditions Monitoring and Optimal Placing of Reactive Power Sources in Distributions Networks
}

\author{
Alexandru Baloi ${ }^{1, a}$, Adrian Pana ${ }^{1, b}$ \\ ${ }^{1}$ Bd. Vasile Parvan, No.2, Timisoara, Romania \\ aalexandru.baloi@et.upt.ro, badrian.pana@et.upt.ro
}

Keywords: distribution network, harmonic conditions, harmonic impedance, capacitor bank, harmonic filter.

\begin{abstract}
Power factor correction in electrical distribution networks can lead to harmonic risk increase. The paper presents a method to anticipate the amplification of network harmonic conditions based on the equivalent network harmonic impedance which can be monitored using virtual instruments. Based on the values of the harmonic impedance and the required reactive power, a mathematical model is implemented through an algorithm and software classes are developed for the solution regarding the type of the reactive power sources (capacitor banks or filters) and the place where it will be installed.
\end{abstract}

\section{Introduction}

Switching on a capacitor bank for shunt reactive power compensation in an electrical distribution network can't be allowed today without checking of the sizing from the condition to avoid the parallel resonance LC between the capacitor bank and the network, due to the increasingly harmonic pollution of the present-day operating conditions of the electrical distribution networks.

It's known that if the parallel LC resonance frequency is close to the frequency of the harmonic currents flowing in the network, these currents will find new closing ways with higher impedances that before the switching of the capacitor bank and that will produce increasing harmonic voltage dropping, and the amplification of harmonic voltage condition, and therefore of harmonic currents [1].

Practically all the transversal elements of the network will be crossed by rms harmonic currents greater that before the switching of the capacitor bank. If the elements of the network corresponding to the $\mathrm{R}$ or $\mathrm{L}$ type of transversal parameters in the equivalent schema can easily support overcurrents, this is not valid for the capacitor bank, which can generally support maximum 30\% overcurrent of the nominal current value, and it means that the situation of impossibility to maintain the capacitor bank in the network can easily appear.

The amplification of the harmonic pollution depends on the value of the capacitance of the capacitor bank installed and on the values of the harmonic currents of the network. Usually result an increase of the voltage total harmonic distortion in the network buses, and the capacitor bank will be crossed by a current having a rms value which can became dangerous from the point of view of thermal stability [1].

That's why before the installation of a capacitor bank in a bus of a network harmonic polluted, the risk evaluation of the increase of the THD voltage values in the bus and on the rms value of the current through the capacitor bank, after its installation, must be done.

The paper propose an algorithm to connect the capacitor banks in the buses of an electrical network or to install harmonic filters if is necessary. The main instrument of the algorithm is the equivalent harmonic impedance of the network which can be monitored in real networks using virtual instruments [2]. The harmonic impedance network is actually the variation in the frequency of direct sequence impedance component seen in a section of that network, section usually considered the point of common coupling (PCC) between the distribution network and the network of a consumer. Most times it is necessary to determine the harmonic impedance of the assembly 
consisting by the distribution network and the network of the consumer. It can be considered that the harmonic impedance seen in a section of the network characterizes its frequency response in that section.

When it comes to monitoring harmonic impedance, its determination must be done by direct measurement [3]. In the literature there are several methods of direct measurement that can be classified into two categories [4] using the transient regimes [5] and the other based on stationary regimes [6].

Knowing the harmonic voltage in the bus, the values of the equivalent network harmonic impedance and the reactive power of the capacitors that will be switched on, the effects of the harmonic conditions amplification can be anticipated and the capacitors for power factor correction must be replaced by harmonic filters providing the same reactive power.

For the present paper, a 10 buses network was modeled using MatLab Simulink in order to get the harmonic conditions (voltage and impedance) for each bus. This values are afterwards used in file like input data for a soft which give the solution of compensation (capacitor bank or filter).

\section{The Algorithm for Reactive Power Compensation}

As we know, connecting a capacitor bank for reactive power shunt compensation in a distribution electrical network harmonically polluted, can lead to the amplification of harmonic voltage. The anticipation of the harmonic voltage amplification and the total harmonic distortion factor of the voltage, in the compensation bus, after the installation of the capacitor bank can be computed as it is demonstrated in [7].

The value of the total harmonic distortion factor is imposed by [8]:

$T H D_{U}<0.08$

Regarding the risk of the capacitor current increase, the manufacturers of the capacitor bank indicate a maximum value of the current through the capacitor:

$I_{C B}<1.3 \cdot I_{n}$

where $I_{C B}$ is the total current through the capacitor bank;

and $I_{n}$ is the nominal current of the capacitor bank.

Under nonsinusoidal conditions, this value can be sometimes overlaid. Considering that in the network there are several buses with different reactive power requested, in order to facilitate the implementation of this condition in the algorithm, the current through the capacitor bank can be monitored by monitoring the value of the total harmonic distortion factor of the current like it follows:

$I_{C B}=\sqrt{I_{C B 1}^{2}+\sum_{k=2}^{\infty} I_{C B k}^{2}}=I_{C B 1} \sqrt{1+\left(T H D_{I_{C B}}\right)^{2}}$

Putting now the condition (2) result:

$$
I_{C B 1} \sqrt{1+\left(T H D_{I_{C B}}\right)^{2}}<1.3 \cdot I_{n}
$$

And considering that the nominal voltage of the capacitor current is the fundamental current, it follows: 


$$
\begin{aligned}
& 1+\left(T H D_{I_{C B}}\right)^{2}<1.69 \\
& \left(T H D_{I_{C B}}\right)^{2}<0.69 \\
& T H D_{I_{C B}}<0.83
\end{aligned}
$$

where $T H D_{I_{C B}}$ is the total harmonic distortion factor of the current;

$I_{C B 1}$ is the fundamental current of the capacitor bank;

$I_{C B k}$ is the harmonic current through the capacitor bank.

The strategic placement methods and planning of harmonic filters are commonly used in harmonic mitigation process [9], [10], but this paper propose an algorithm for optimal planning of capacitor banks and to replace it with harmonic filters only in certain conditions for reducing the costs. The main instrument of the presented algorithm is a sensitivity factor, computed for each bus where a transversal compensation will be done. Considering the existing harmonic conditions (harmonic voltage and harmonic impedance which can be determined) and the compensation reactive power level, the sensitivity factor is defined like it follows:

$$
S_{i}=\frac{T H D_{U_{i}^{*}}-T H D_{U_{i}}}{Q_{i}}
$$

where:

$T H D_{U_{i}^{*}}$ - the total harmonic distortion voltage in bus $i$, after the installation of the capacitor bank, which can be anticipated [7];

$T H D_{U_{i}}$ - the total harmonic distortion voltage in bus $i$, before the installation of the capacitor bank;

$Q_{i}$ - the reactive power of the capacitor bank in bus $i$.

The sensitivity factor is a measure of the voltage distortion amplification due to the capacitor bank installation, and it value must be as lower as possible. The utility of the sensitivity factor defined by (8), is the information about the risk for capacitor bank overstressing and the priorities regarding the installation of new capacitor bank or new harmonically loads. These installations will be done in the buses having a lower sensitivity factor, because in those buses we have a reserve of voltage distortion amplification.

After the sensitivity factor calculus for each bus, an ascending sort is done and in the first bus, the corresponding capacitor bank is installed. If at least one of the conditions (1), (7) is not accomplished, the capacitor bank will be replaced by harmonic filters which produce the same power factor correction. After this installation the procedure of computing the sensitivity factor for the rest of the buses in the new configuration continues until the last bus.

The input data read from a text file are general data:

$n$ - the number of buses;

$m$ - the number of harmonics considerate;

$T H D_{U \max }$ - the accepted value for total harmonic distortion voltage (0.08);

$T H D_{I_{C B \max }}$ - the accepted value of the current through the capacitor bank (0.83);

and harmonic values corresponding to each bus:

$X_{C_{i, k}}$ - the harmonic reactance of the capacitor bank that will be installed in bus $\mathrm{i}$;

$U_{i, k}$ - the harmonic voltage of bus i, before the installation of the capacitor bank;

$Z_{i, k}$ - the harmonic impedance seen in bus i, before the installation of the capacitor bank.

All the electric quantities used in this algorithm are complex numbers.

In Fig. 1 is presented the flowchart of the algorithm. 


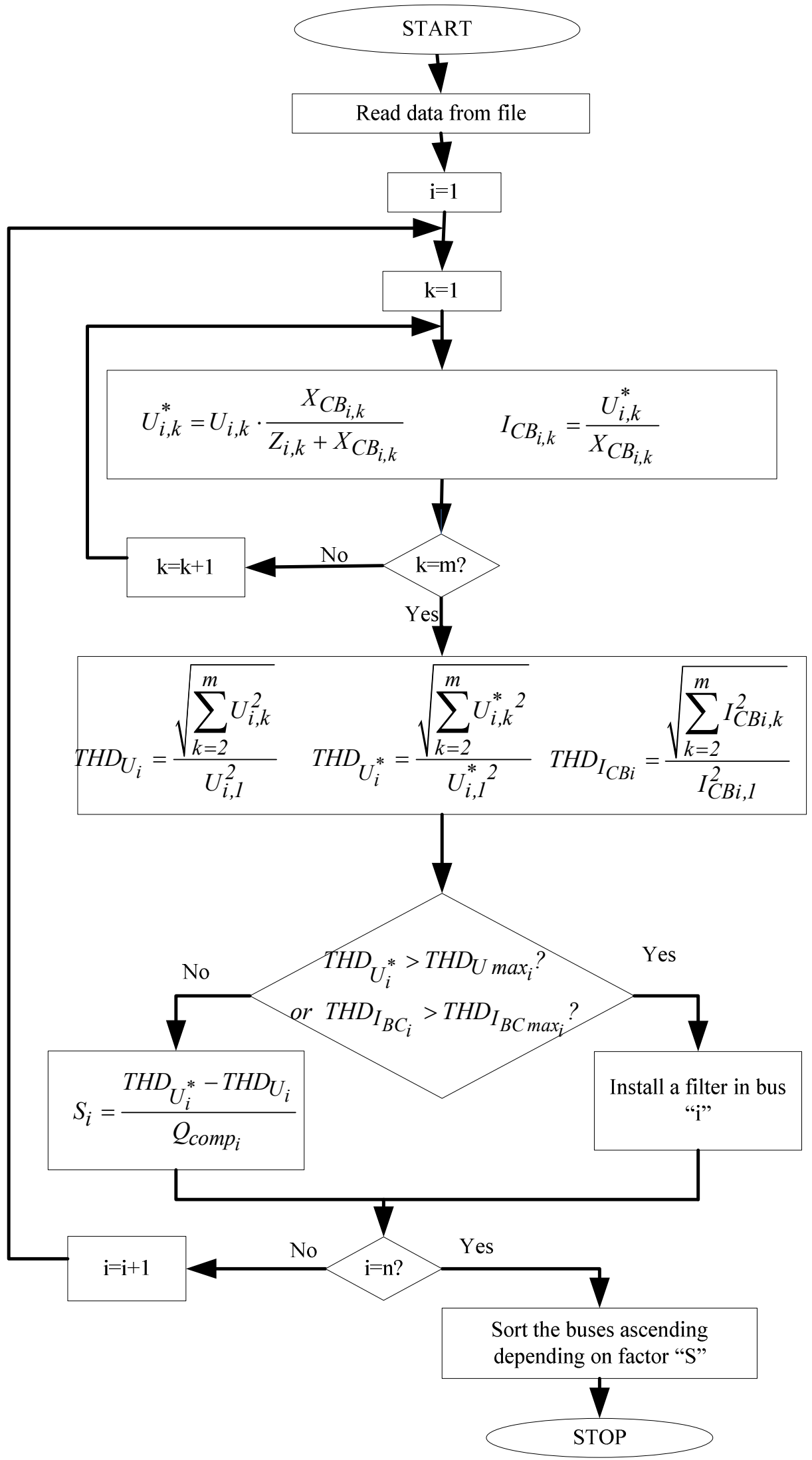

Fig. 1. The flowchart of the algorithm 


\section{Software Solutions for Reactive Power Compensation}

The distribution network presented in Fig.2, having 10 load buses and capacitor banks for power factor correction installed in each bus is considered.

The operating conditions regarding the active and reactive power of the loads, the reactive power of the capacitor banks and the magnitude of the harmonic current sources considerate in each bus are presented in Table I.

Also, the lengths of the supplying electrical cables are presented for both $20 \mathrm{kV}$ and $0.4 \mathrm{kV}$ networks.

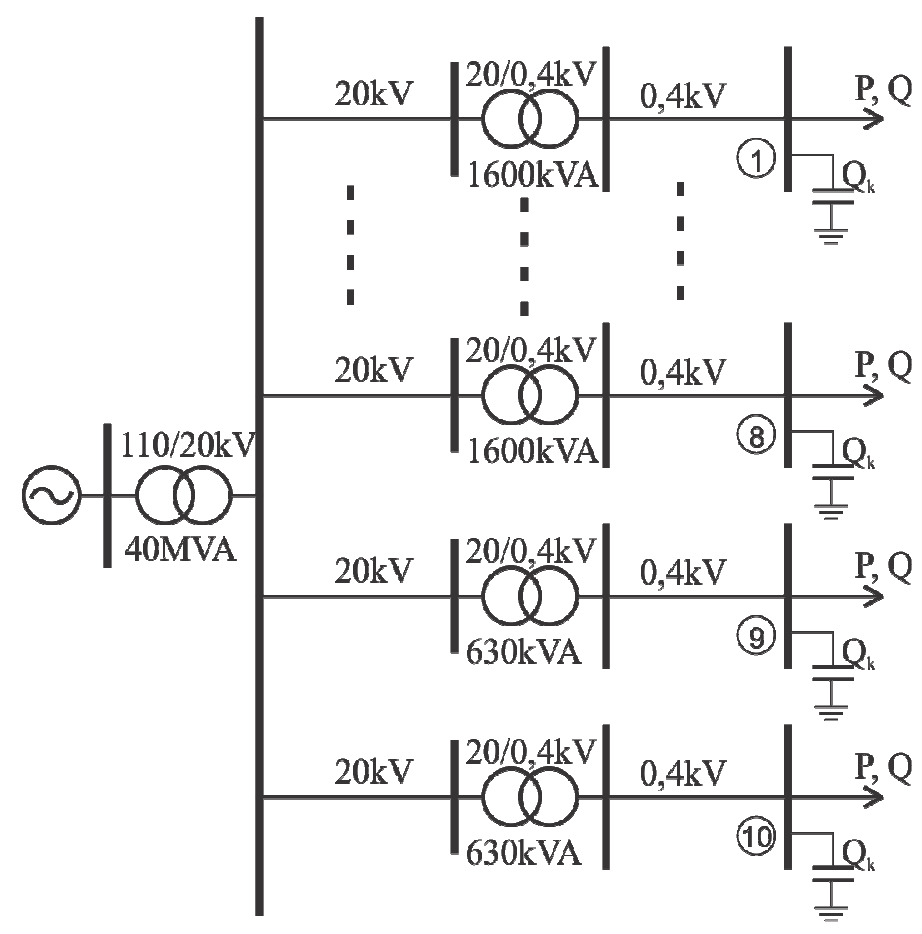

Fig.2. The configuration of the electrical network used for the application.

Table I. The operating conditions of the network.

\begin{tabular}{|c|c|c|c|c|c|c|c|c|}
\hline Bus & $\mathrm{P}[\mathrm{kW}]$ & $\mathrm{Q}[\mathrm{kVAr}]$ & $\mathrm{Q}_{\mathrm{cb}}[\mathrm{kVAr}]$ & $\mathrm{I}_{9}[\mathrm{~A}]$ & $\mathrm{I}_{7}[\mathrm{~A}]$ & $\mathrm{I}_{11}[\mathrm{~A}]$ & $\mathrm{L}_{20 \mathrm{kV}}[\mathrm{km}]$ & $\mathrm{L}_{0,4 \mathrm{kV}}[\mathrm{km}]$ \\
\hline 1 & 820 & 720 & 450 & 90 & 85 & 95 & 0,4 & 0,1 \\
\hline 2 & 870 & 740 & 325 & 85 & 85 & 85 & 0,25 & 0,12 \\
\hline 3 & 780 & 700 & 450 & 110 & 120 & 90 & 0,2 & 0,4 \\
\hline 4 & 810 & 740 & 450 & 85 & 80 & 100 & 0,35 & 0,09 \\
\hline 5 & 790 & 720 & 450 & 80 & 75 & 70 & 0,25 & 0,2 \\
\hline 6 & 750 & 640 & 250 & 70 & 95 & 80 & 0,2 & 0,5 \\
\hline 7 & 760 & 680 & 400 & 70 & 70 & 70 & 0,3 & 0,25 \\
\hline 8 & 740 & 660 & 500 & 80 & 90 & 80 & 0,22 & 0,3 \\
\hline 9 & 520 & 340 & 225 & 60 & 75 & 85 & 0,1 & 0,6 \\
\hline 10 & 450 & 280 & 150 & 90 & 80 & 80 & 0,15 & 0,1 \\
\hline
\end{tabular}

MatLab Simulink was used for having the harmonic voltages and the harmonic impedances before the installation of the capacitor banks in each bus, necessary to determine the sensitivity factor. The results for harmonic impedance obtained by MatLab Simulink (Fig.3), for each harmonic range of the current flowing in the network, are used like complex number input data in the algorithm. 


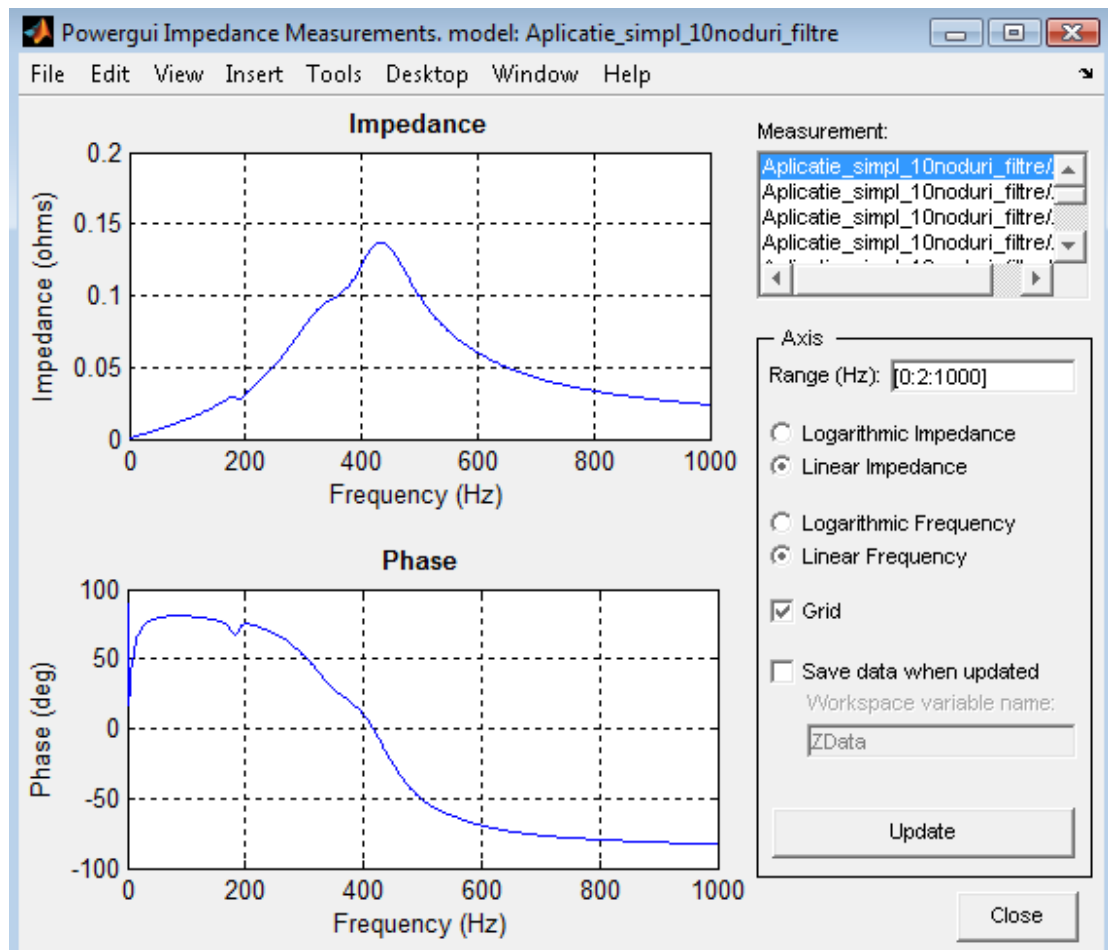

Fig.3. The harmonic impedance in MatLab Simulink

Software was developed using C\# and the input data (complex numbers) are read from a txt file. The corresponding interface created is presented in Fig.4, where Buses Harmonic Voltage and Harmonic impedance seen in the buses are 10x4 matrix comprising complex numbers taken from MatLab Simulink. Harmonic reactance of the capacitor banks that will be connected is also a 10x4 matrix. Three harmonics current were considerate $(7,9,11)$ and the fundamental.

\begin{tabular}{l} 
Algorithm \\
$\begin{array}{l}\text { File Options Help } \\
\text { Main Data } \\
\text { Number of buses }\end{array}$ \\
Accepted Voltage THD \\
Buses Harmonic Voltage \\
\hline Harmonic impedance seen in the buses
\end{tabular}

Fig.4. The software interface for input data.

The solution of the algorithm for the present application is represented by Buses List sorted by the sensitivity factor (Fig.5). Only in buses 2 and 3 the reactive power shunt compensation must be done using filters, in the rest of the network the conditions imposed by the proposed algorithm are accomplished. For bus 2, both the conditions regarding the total harmonic distortion factor (voltage and capacitor current) are unfulfilled, and for bus 3 only the condition regarding the voltage total harmonic distortion factor is unfulfilled. 


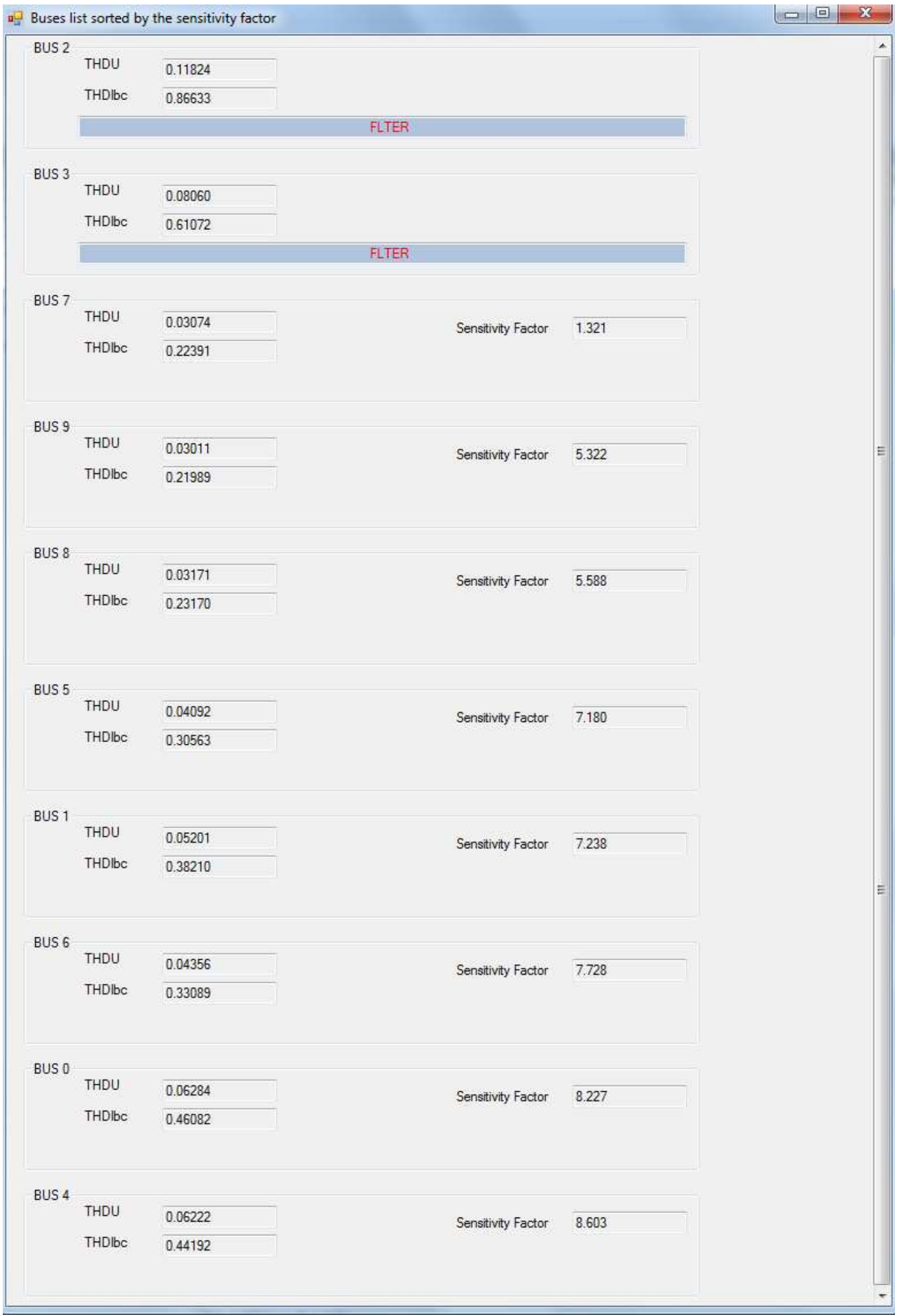

Fig.5. The software interface for the results of the algorithm. 


\section{Conclusions}

The paper presents an algorithm for power factor correction an electrical distribution network which is based on a quickly method for evaluating the effects of a transversal impedance installation in the presence of harmonics. The main instrument of this method is the harmonic impedance seen in the respective section of the network.

The mathematical model allows the anticipation of both the harmonic voltage level in the bus and the current trough the shunt capacitor banks which will be installed.

The THD of the capacitor current can be considered also as an indicator of the amplification of the harmonic current on the shunt elements.

The method allows identifying the buses where unwanted effects can appear when a capacitor bank is installed and where it must be replaced by harmonic filter. A classification of the buses depending on the sensitivity factor is proposed. This classification can be useful regarding the installation of new capacitor banks or new harmonic loads.

\section{Acknowledgement}

This paper was supported by the project "Develop and support multidisciplinary postdoctoral programs in primordial technical areas of national strategy of the research - development innovation" 4D-POSTDOC, contract nr. POSDRU/89/1.5/S/52603, project co-funded from European Social Fund through Sectorial Operational Program Human Resources 2007-2013.

\section{References}

[1] J., Arrillaga, D., A., Bradley, P., S., Rodger, "Power Systems Harmonics", John Wiley \& Sons, New York, 1985.

[2] A., Baloi, A., Pana, F., Molnar-Matei, "Contributions on Harmonic Impedance Monitoring in Smart Grids Using Virtual Instruments", Proceedings of the $2^{\text {nd }}$ IEEE PES International Conference on Innovativ Smart Grid Technologies, Europe 2011, Manchester, (2011), ID:40.

[3] J., Barros, Matilde de Apraiz, R. I., Diego, "A virtual measurement instrument for electrical power quality analysis using wavelets", Measurement, No. 42, 2009, pp. 298-307, Elsevier.

[4] W., Xu, E.E., Ahmed, X., Yhang, X., Liu, "Measurement of Network Harmonic Impedances: Practical Implementation Issues and Their Solutions", IEEE Transactions on Power Delivery, Vol. 17, No. 1, January 2002.

[5] A., Robert, T., Deflandre, "Guide for assessing the network harmonic impedance", WG CC02, Electra, August, 1996.

[6] A., de Oliveira, J.C., de Oliveira, J.W., Resende, M.S., Miskulin, "Practical approaches for AC system harmonic impedance measurements", IEEE Transactions on Power Delivery, Vol. 6, No. 10, October 1991.

[7] A., Pana, A., Baloi, "A quickly method to estimate harmonic conditions changes in a bus of an electrical network, as a result of transversal impedance installation", Proceedings of the $8^{\text {th }}$ WSEAS International Conference on Power Systems, Santander, Spain, (2008), 89-94.

[8] *** EN 50160, Voltage characteristics of electricity supplied by public distribution systems, CENELEC, Brussels, November 1999.

[9] H. L., Ginn, L. S., Czarnecki, "An Optimization Based Method for Selection of Resonant Harmonic Filter Branch Parameters," IEEE Transactions on Power Delivery, Vol. 21, No. 3, pp. 1445-1451, Jul. 2006.

[10] Y. P. Chang, and C. J. Wu, "Optimal Multiobjective Planning of Large-Scale Passive Harmonic Filters Using Hybrid Differential Evolution Method Considering Parameter and Loading Uncertainty," IEEE Transactions on Power Delivery, Vol. 20, No. 1, pp. 408-416, Jan. 2005. 